\title{
Relevância dos Índices Ómega-3 e Razão Ómega-6 I Ómega-3 na Prevenção do Défice Cognitivo
}

\section{Relevance of Omega-3 and Omega-6 / Omega-3 Ratio in Preventing Cognitive Impairment}

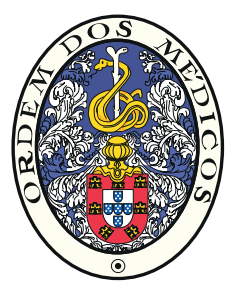

\author{
Belina NUNES $\square^{1}$, Cristina PINHO ${ }^{1}$, Cláudia SOUSA ${ }^{1}$, Alan Rocha MELO',2, Narcisa BANDARRA ${ }^{3}$, Maria Carolina SILVA ${ }^{4}$
} Acta Med Port 2017 Mar;30(3):213-223 - https://doi.org/10.20344/amp.7970

\section{RESUMO}

Introdução: A relevância epidemiológica e social da demência e do défice cognitivo ligeiro aumenta com a esperança de vida e prevalência das doenças metabólicas. O reconhecimento da importância dos nutrientes no funcionamento cognitivo perspectiva novas estratégias preventivas. Neste trabalho analisamos a associação entre os valores do índice ómega-3 e da razão ómega-6 / ómega-3 e desempenho cognitivo/ défice cognitivo ligeiro.

Material e Métodos: Selecionamos uma amostra aleatória de utentes de um centro de saúde com idade $\geq 55$ anos, que foram inquiridos sobre hábitos alimentares e sujeitos a avaliação neuropsicológica, incluindo o Montreal Cognitive Assessment, e determinando-se os índices indicados.

Resultados: Os 90 participantes tinham em média 64 anos ( $\mathrm{dp}=5,6), 71,1 \%$ quatro anos de escolaridade e $40 \%$ mais de um factor de risco vascular. Os limites dos valores de ómega-3 e da razão ómega-6 / ómega-3 foram respectivamente 2,90 - 9,79 (média = 6,20; $d p=1,50)$ e 1,14 - 2,95 (média = 2,12; dp = 0,39). A pontuação no Montreal Cognitive Assessment variou entre $10-29$ (média = 22,0; $\mathrm{dp}=4,5$ ), aumentando em média 1 - 2 pontos para valores de ómega-3 acima da média e uma razão ómega-6 / ómega-3 2 (Grupo 1) e diminuindo 5 - 6 pontos para um valor de ómega-3 abaixo da média e a mesma razão ómega- 6 / ómega-3 (Grupo 2). A prevalência de défice cognitivo ligeiro foi 25,6\% (IC 95\%:16,4 - 34,7\%), variando entre 12,5\% e 83,3\% nos dois grupos mencionados.

Discussão: Este estudo preliminar é um dos primeiros a analisar a repercussão do índice ómega-3 e da razão ómega-6 / ómega-3 na função cognitiva, considerando adicionalmente o perfil clínico e consumo alimentar dos participantes.

Conclusão: A associação encontrada sugere que o índice ómega-3 e a razão ómega-6 / ómega-3 podem ser biomarcadores importantes na prevenção da deterioração cognitiva.

Palavras-chave: Ácidos Gordos Ómega-3; Ácidos Gordos Ómega-6; Envelhecimento; Perturbações da Cognição

\section{ABSTRACT}

Introduction: The epidemiological and social relevance of dementia and mild cognitive impairment increases with life expectancy and prevalence of metabolic disorders. The recognition of an important role for dietary nutrients on cognitive functioning opens new preventive strategies. This work analyses the association between erythrocyte omega-3 index and omega- 6 / omega-3 ratio and cognitive performance/ mild cognitive impairment.

Material and Methods: We selected a random sample of individuals registered at a health centre aged $\geq 55$ years. Participants were asked about their alimentary habits, erythrocyte indexes were determined and neuropsychological evaluation included the Montreal Cognitive Assessment and a comprehensive test battery.

Results: Mean age of 90 participants was 64 years $(\mathrm{sd}=5.6), 71.1 \%$ had four years of education and $40 \%$ of them had more than one vascular risk factor. Limits for omega-3 index and omega-6 / omega-3 ratio were $2.90-9.79$ (mean = 6.20; sd = 1.50) and $1.14-2.95$ $($ mean $=2.12 ; s d=0.39)$, respectively. Montreal Cognitive Assessment scores ranged from 10 to 29 (mean $=22 ; s d=4.5)$, increasing on average 1 - 2 points for above average omega-3 values and an omega- 6 / omega-3 ratio $\leq 2$ (Group 1) and decreasing 5 - 6 points for omega-3 below mean values and the same omega- 6 / omega-3 ratio (Group 2). Prevalence of mild cognitive impairment was $25.6 \%$ (95\% Cl: $16.4-34.7)$, ranging from $12.5 \%$ to $83.3 \%$ in the two groups above.

Discussion: This preliminary study is one of the first analyzing the repercussion of omega- 3 index and omega- 6 / omega-3 ratio on cognitive functioning, considering additionally the participant clinical and dietary profiles.

Conclusion: The association found suggests that omega- 3 and omega- 6 / omega- 3 ratio could be important biomarkers in the prevention of cognitive impairment.

Keywords: Aging; Cognition Disorders; Fatty Acids, Omega-3; Fatty Acids, Omega-6

\section{INTRODUÇÃO}

A demência e o défice cognitivo ligeiro (DCL) são entidades clínicas com importância crescente face ao enveIhecimento da população e devido ao seu elevado impacto social, psicológico e económico para os doentes, família e sociedade em geral. A investigação na área das demências tem, nos últimos anos, direccionado recursos significativos para o estudo do DCL, a sua diferenciação do en- velhecimento normal e a conversão em demência. A sua prevalência em indivíduos de idade superior a 65 anos é de $12 \%-15 \%$, com uma taxa de conversão anual de DCL amnésico em doença de Alzheimer (DA) de cerca de 10 a $15 \%$, contrastando com taxas não superiores a $1 \%$ a $2 \%$ numa população de indivíduos normais da mesma idade..$^{1-6}$ Em Portugal a prevalência de DCL entre os 55 - 79 anos foi

\footnotetext{
1. Associação de Estudos de Demência. Porto. Portugal.

2. Centro de Saúde de Lousada. Lousada. Portugal.

3. Divisão de Aquacultura e Valorização. IPMA - Instituto Português do Mar e da Atmosfera. Lisboa. Portugal.

4. Instituto de Ciências Biomédicas de Abel Salazar. Universidade do Porto. Porto. Portugal.

$\triangle$ Autor correspondente: Belina Nunes. bnunes@weblink.pt

Recebido: 22 de junho de 2016 - Aceite: 01 de dezembro de 2016 | Copyright @ Ordem dos Médicos 2017
} 
de $12,3 \%$ em 2003 - 2004. ${ }^{7}$ Destes valores se infere a relevância do conceito e a necessidade de manter este estrato populacional sob vigilância regular.

É considerado indiscutível que os maiores benefícios na desaceleração da prevalência da demência decorrerão de intervenções precoces, sendo os grupos de doentes com DCL, cada vez mais a população alvo de diversos ensaios clínicos. Embora não exista, até esta data, nenhum medicamento ou terapia comprovadamente eficaz para impedir a evolução do DCL para demência, uma das linhas de investigação mais promissoras tem sido a que explora os efeitos da alimentação e dos seus diferentes nutrientes na cognição. A alimentação de tipo mediterrânico - com consumo de peixe, vegetais, fruta fresca, azeite e nozes - parece ter um papel protector, assim como tem sido evidenciado o benefício da suplementação da dieta com vitamina B12, B6, ácido fólico, antioxidantes e ácidos gordos polinsaturados ómega-3 (n-3) (PUFA). De entre os diversos agentes investigados, têm mostrado maiores benefícios neuroprotectores os ómega-3 PUFA (DHA: ácido docosahexanóico e EPA: ácido eicosapentanóico), a fosfatidilserina, o ácido fólico, as vitaminas E e B12 e a Ginkgo biloba. ${ }^{8-14}$

$\mathrm{O}$ índice ómega-3 tem vindo a ser reconhecido como um relevante marcador do risco de doença cardiovascular e começa recentemente a ser avaliado como possível marcador de disfunção cognitiva. Deste modo, um índice ómega-3 $\geq 8 \%$ associa-se a maior protecção cardíaca, enquanto que $\leq 4 \%$ se associa a risco de doença coronária, ${ }^{15}$ sendo os ácidos gordos ómega-3 reconhecidos como nutrientes essenciais para promover um envelhecimento saudável. ${ }^{16}$ $\mathrm{Na}$ avaliação clínica de DCL é habitualmente efectuado estudo laboratorial extenso mas não é prática corrente a determinação do índice ómega-3 ou a razão ómega-6 / ómega-3 (n-6 / n-3). No entanto, estudos recentes revelam uma associação entre a razão ómega-6 / ómega-3 e demência ou declínio cognitivo, com uma diminuição de ingestão de n-3 PUFA ou aumento do consumo de ómega-6 (n-6) PUFA na alimentação podendo acelerar o declínio cognitivo entre 10 e $80 \% .^{17}$

Na dieta humana ancestral o índice $n-6 / n-3$ seria de $1: 1$, tendo vindo a aumentar nas sociedades industrializadas para valores muitíssimo superiores, tendo sido reportados níveis de 15:1 ou mesmo de 25:1 na dieta da população ocidental. A síntese de DHA no cérebro é muito limitada e, por esse motivo, os efeitos dos n-3 PUFA sobre a cognição são expectavelmente superiores em dietas com predomínio de DHA, do que em dietas contendo os seus precursores. ${ }^{17,18}$ Alguns estudos revelaram, por exemplo, valores mais baixos do índice n-3 em doentes com doença de Alzheimer do que nos controlos saudáveis. ${ }^{19,20} \mathrm{Em}$ modelos animais de DA, a suplementação da dieta com DHA por um período de três mese ${ }^{21}$ ou dieta com uma razão $n-6$ / n-3 baixa ${ }^{22,23}$ diminui a razão n-6/n-3 no cérebro e baixa os níveis cerebrais de $A \beta$ e tau insolúvel, principais marcadores patológicos da DA. Num estudo recente a suplementação com DHA num grupo de doentes com deterioração cognitiva revelou benefícios ao fim de seis meses em comparação com o grupo placebo. ${ }^{24}$ Benefícios de uma dieta enriquecida em n-3 PUFA (vs n-6 PUFA) foram também demonstrados na evolução de marcadores de risco aterosclerótico (perfil lipídico, citoquinas inflamatórias, parâmetros reológicos, homocisteína) ou na estabilização de placas ateroscleróticas carotídeas e coronárias. ${ }^{25-27}$

Vários estudos populacionais, incluindo na população portuguesa, ${ }^{7}$ têm paralelamente demonstrado que os clássicos factores de risco cardiovascular são também proeminentes marcadores de risco de demência, mesmo no respeitante às formas neurodegenerativas não-vasculares (eg. Alzheimer), a fazer supor a existência de mecanismos fisiopatogénicos partilhados ou sinérgicos.

Os resultados descritos sugerem que uma baixa razão n-6 / n-3 na dieta obtida através de um maior consumo de n-3 PUFA, ou um menor aporte relativo de n-6 PUFA pode ter efeitos benéficos importantes na manutenção da função cognitiva e prevenção da demência. O objectivo deste estudo é analisar a relação entre o desempenho cognitivo/ DCL e valores sanguíneos do índice ómega-3 e da razão ómega-6 / ómega-3, considerando adicionalmente o perfil clínico e alimentar dos participantes.

\section{MATERIAL E MÉTODOS \\ Amostra}

Foi selecionada uma amostra aleatória de utentes do Centro de Saúde do concelho de Lousada baseada nos seguintes critérios: inscrição activa, número par e idade superior a 55 anos. Foram excluídos os utentes com

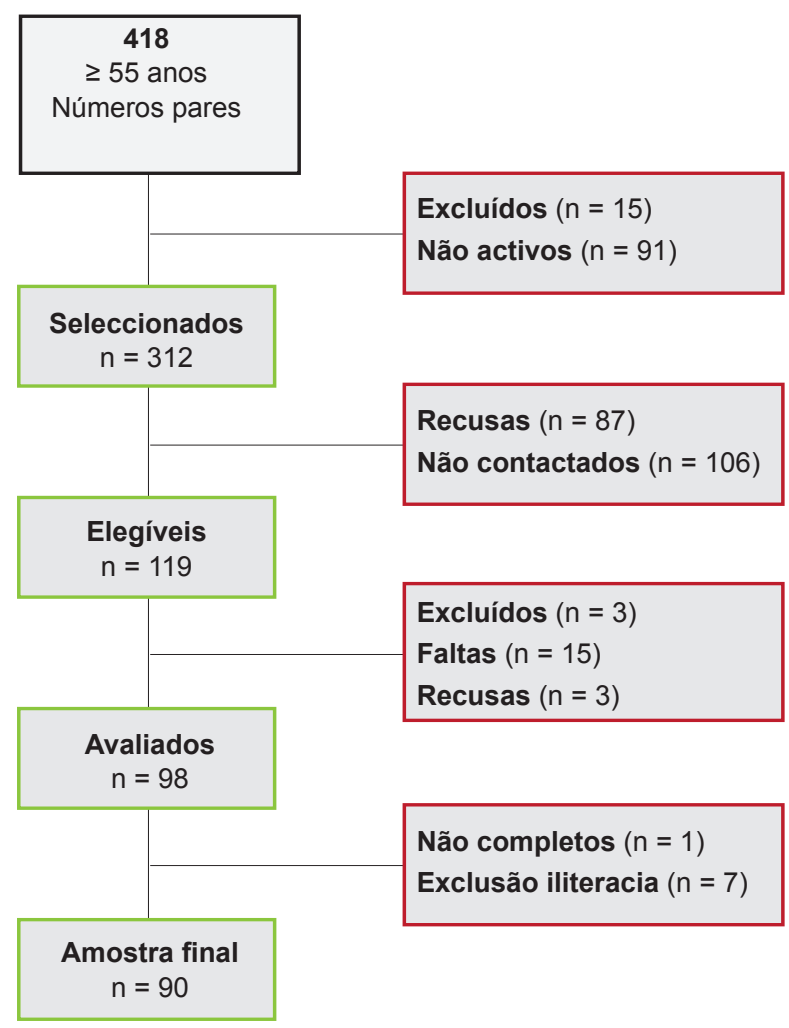

Figura 1 - Processo de recrutamento da amostra Lista de utentes: $\mathrm{n}=1872$ 
história de abuso de álcool ou outras substâncias psicotrópicas tendo por base a consulta dos ficheiros clínicos (Fig. 1). O recrutamento foi feito através de contacto telefónico, tendo terminado quando foi atingida uma dimensão amostral de aproximadamente 120 participantes, considerando-se este número adequado para uma análise de regressão múltipla envolvendo 10 variáveis preditoras.

\section{Procedimento}

Após aprovação do estudo pela direcção do centro de saúde, os objectivos e procedimentos do estudo foram previamente explicados a cada um dos participantes e/ou cuidadores, sendo assinado um consentimento informado. Da avaliação de cada participante constou:

a) Entrevista semi-estruturada para recolher dados sócio-demográficos, história médica com estudo analítico detalhado e recolha da toma actual de fármacos e suplementos vitamínicos;

b) AUDIT modificado (seis itens) com limites entre 0 - 21 pontos, usado apenas para determinar os hábitos de consumo de álcool ${ }^{28}$;

c) Escala de Queixas Subjectivas de Memória (10 itens), com ponto de corte $>3^{29,30}$;

d) Montreal Cognitive Assessment (MoCA) adoptando como pontos de corte para definir compromisso cognitivo valores de 1 desvio padrão abaixo da média segundo a idade e escolaridade (idades 55 - 64 e $\geq 65$ anos e escolaridade 1 - 4, 5 - 9, 10 - 12 e > 12 anos. ${ }^{31,32}$ Em relação aos vários domínios que integram o MoCA, usamos os seguintes critérios para definir compromisso cognitivo: i) Visuo-Espacial/ Executivo (inclui as provas de Trail Making Test, Cubo e Relógio) a totalidade dos subtestes com pontuação $\geq 3$ pontos; ii) Nomeação se $\leq 1$ ponto; ii) Atenção (Memória de Dígitos, Série de letras e Subtracção) subtestes < 3 pontos; iv) Linguagem (Repetição e Fluência verbal) soma dos subtestes $\leq 1$ ponto; v) Abstracção $\leq 1$ ponto e sem sucesso no item exemplo; vi) Memória foi considerada presença de compromisso quando $\leq 2$ pontos; vii) Orientação compromisso quando $<3$;

e) Bateria de testes neuropsicológicos incluindo: i) Teste do Relógio, ${ }^{33}$ com o sistema quantitativo de cotação de 18 pontos $^{34}$; ii) Teste de fluência semântica (animais) e fonémica (letra ' $P$ ' $)^{35,36}$; iii) Teste Stroop ${ }^{37,38}$; iv) Hopkins Verbal Learning Test- HVLT-R ${ }^{39}$; v) Subteste Memória de Dígitos da Wechsler Memory Scale - WMS-III ${ }^{40}$; vi) Subteste Pesquisa de Símbolos da Wechsler Adult Inteligence Scale ${ }^{41}$; vii) Trail Making Test - Parte A e B. ${ }^{42,43}$ À semelhança do MoCA, os resultados obtidos nos testes cognitivos foram posteriormente agrupados em domínios cognitivos (Atenção: Memória de Dígitos (WAIS-III), Pesquisa de Símbolos (WAIS-III), Trail Making Test (parte A), Teste Stroop ( $1^{a}$ parte - Palavra e $2^{a}$ parte Cor); Memória: Hopkins Verbal Learning Test; Funções executivas: Teste do Relógio, Teste Stroop ( $3^{a}$ parte cor-palavra e interferência), Trail Making Test (parte B) e Teste de fluência fonémica (letra ' $P$ '); Linguagem: Tes- te de fluência semântica ('animais'); Visuo-construção: Cópia do Teste do Relógio) de modo a melhor definir o perfil cognitivo da amostra;

f) Escala de Actividades Instrumentais de Vida Diária Instrumental Activities of Daily Living Scale (IADL), com ponto de corte $\geq 9^{44,45}$;

g) Escala Hospitalar de Ansiedade e Depressão Hospital Anxiety and Depression Scale (HADS), ausência - < 8; ligeira 8 - 0; moderada > 1046;

h) Questionário alimentar baseado na frequência do consumo de peixe (bacalhau, peixe magro, peixe gordo, peixe em conserva), carne (branca, vermelha, fumada), frutos secos (nozes, amêndoas, avelãs), lacticínios, hortaliças e legumes, numa escala de 0 - nunca a 7 diariamente.

A avaliação cognitiva foi realizada por duas neuropsicólogas (C. P. e C. S.) no centro de saúde. Os participantes fizeram ainda colheitas de sangue para a determinação de índice ómega-3 e razão ómega-6 / ómega-3, tendo sido recolhido $10 \mathrm{~mL}$ de sangue venoso. Após repouso, procedeu-se à separação do plasma por centrifugação a 700 g (2500 rpm) durante $10 \mathrm{~min}$. O plasma resultante foi eliminado. O pellet de eritrócitos foi lavado com igual volume de soro fisiológico (solução aquosa de $\mathrm{NaCl}$ a $0,9 \%$ ). Tendo-se procedido a centrifugação a $700 \mathrm{~g}$ durante $5 \mathrm{~min}$. O pellet foi novamente lavado. Recolhendo-se $0,5 \mathrm{~mL}$ de pellet para um tubo identificado e rolhado que se conservou a $-80^{\circ} \mathrm{C}$ até posterior análise. Os eritrócitos obtidos foram usados na análise dos ácidos gordos dos fosfolípidos seguindo a metodologia referida em estudo prévio. ${ }^{47}$

Os valores destes índices foram dicotomizados seguindo os critérios: para o índice ómega-3 considerou-se a média amostral e para a razão ómega-6 / ómega-3 o valor 2 referido como valor limite para um balanço adequado. ${ }^{48}$

\section{Análise estatística}

Para estudar a relação entre a avaliação do desempenho cognitivo (MoCA) e as variáveis definidoras do perfil sócio-demográfico, funcionalidade, comorbilidades, medicação e índices ómega-3 e razão ómega-6 / ómega-3 utilizaram-se modelos de análise de variância (ANOVA) e comparações múltiplas pelo teste de Scheffé, após verificação dos pressupostos de normalidade (teste de Kolmogorov-Smirnov) e igualdade de variâncias (teste de Levene), admitindo um valor de $p>0,01$ para verificação dos pressupostos; no caso de variáveis ordinais estudou-se a existência de uma tendência linear e/ou quadrática nas médias. Para avaliar a relação entre o consumo alimentar, os valores sanguíneos dos eritrócitos e o desempenho cognitivo foi usado o coeficiente de correlação linear de Pearson. Para estudar a associação entre as variáveis já mencionadas e a prevalência de compromisso cognitivo no MoCA foi utilizado o teste do qui-quadrado de Pearson ou o teste exacto de Fisher no caso de variáveis dicotómicas; para variáveis ordinais testou-se a tendência linear nas proporções. Este procedimento foi repetido para o compromisso cognitivo nos vários domínios que integram o MoCA. 
Tabela 1 - Descrição dos participantes, desempenho cognitivo (MoCA) e prevalência de compromisso cognitivo segundo as características sócio-demográficas e avaliação da funcionalidade, depressão e ansiedade

\begin{tabular}{|c|c|c|c|c|c|c|c|}
\hline \multirow{2}{*}{ Características } & \multicolumn{2}{|c|}{ Participantes } & \multicolumn{2}{|c|}{ Avaliação cognitiva } & \multicolumn{3}{|c|}{ Compromisso cognitivo } \\
\hline & $\mathrm{n}$ & $\%$ & Média (dp) & $p \dagger$ & $\mathrm{n}$ & Prevalência (\%) & $p \ddagger$ \\
\hline \multicolumn{8}{|l|}{ Género } \\
\hline Homens & 44 & 48,9 & $22,8(4,2)$ & \multirow[t]{2}{*}{0,12} & 8 & 18,2 & 0,12 \\
\hline Mulheres & 46 & 51,1 & $21,3(4,7)$ & & 15 & 32,6 & \\
\hline \multicolumn{8}{|l|}{ Idade (anos) } \\
\hline $55-59$ & 21 & 23,3 & $24,1(3,7)$ & \multirow{4}{*}{$\begin{array}{l}0,068 \\
0,019\end{array}$} & 4 & 19,0 & 0,63 \\
\hline $60-64$ & 26 & 28,9 & $22,0(4,1)$ & & 7 & 26,9 & 0,76 \\
\hline $65-69$ & 27 & 30,0 & $20,8(4,8)$ & & 9 & 33,3 & \\
\hline$\geq 70$ & 16 & 17,8 & $21,2(4,9)$ & & 3 & 18,8 & \\
\hline \multicolumn{8}{|l|}{ Estado civil } \\
\hline Casado & 78 & 86,7 & $21,8(4,6)$ & \multirow[t]{2}{*}{0,30} & 20 & 25,6 & 0,96 \\
\hline Solteiro / viúvo / divorciado & 12 & 13,3 & $23,3(3,6)$ & & 3 & 25,0 & \\
\hline \multicolumn{8}{|l|}{ Educação (anos) } \\
\hline $1-3$ & 12 & 13,3 & $17,0(4,3)$ & \multirow{3}{*}{$\begin{array}{l}0,001 \\
0,001\end{array}$} & 8 & 66,7 & 0,002 \\
\hline 4 & 64 & 71,1 & $22,0(3,9)$ & & 12 & 18,8 & 0,014 \\
\hline$\geq 5$ & 14 & 15,6 & $26,3(2,0)$ & & 3 & 21,4 & \\
\hline \multicolumn{8}{|l|}{ Situação profissional } \\
\hline Activo & 22 & 24,4 & $21,9(4,8)$ & \multirow{3}{*}{0,17} & 5 & 22,7 & \\
\hline Desempregado & 12 & 13,3 & $24,3(3,0)$ & & 2 & 16,7 & 0,65 \\
\hline Reforma / pensão & 56 & 62,2 & $21,6(4,5)$ & & 16 & 28,6 & \\
\hline \multicolumn{8}{|l|}{ Sector profissional } \\
\hline Quadros / empresários & 16 & 17,8 & $24,6(3,5)$ & \multirow{4}{*}{0,010} & 3 & 18,8 & \\
\hline Produção / indústria & 42 & 46,7 & $22,0(3,9)$ & & 9 & 21,4 & 0,56 \\
\hline Serviços & 9 & 10,0 & $22,8(5,1)$ & & 3 & 33,3 & \\
\hline Não qualificados & 23 & 25,6 & $19,9(5,0)$ & & 8 & 34,8 & \\
\hline \multicolumn{8}{|l|}{ Situação residencial } \\
\hline A viver com família & 84 & 93,3 & $21,9(4,5)$ & \multirow[t]{2}{*}{0,63} & 21 & 25,0 & 0,65 \\
\hline A viver sozinho & 6 & 6,7 & $22,8(3,7)$ & & 2 & 33,3 & \\
\hline \multicolumn{8}{|l|}{ Actividade diária } \\
\hline Sim & 70 & 77,8 & $22,2(4,5)$ & \multirow[t]{2}{*}{0,46} & 16 & 22,9 & 0,27 \\
\hline Não & 20 & 22,2 & $21,4(4,6)$ & & 7 & 35,0 & \\
\hline \multicolumn{8}{|l|}{ Queixas subjectivas memória } \\
\hline Presença & 57 & 63,3 & $21,9(4,6)$ & \multirow[t]{2}{*}{0,85} & 16 & 28,1 & 0,47 \\
\hline Ausência & 33 & 36,7 & $22,1(4,4)$ & & 7 & 21,2 & \\
\hline Funcionalidade - IADL & & & & & & & \\
\hline Independência & 83 & 92,2 & $22,3(4,5)$ & 0,027 & 18 & 21,7 & 0,004 \\
\hline Dependência & 7 & 7,8 & $18,4(2,2)$ & & 5 & 71,4 & \\
\hline Depressão (escala HADS) & & & & & & & \\
\hline Moderada & 4 & 4,4 & $20,0(6,2)$ & 0,27 & 3 & 75,0 & 0,047 \\
\hline Ligeira & 12 & 13,3 & $20,5(4,8)$ & 0,12 & 4 & 33,3 & 0,022 \\
\hline Ausente & 74 & 82,2 & $22,4(4,3)$ & & 16 & 21,6 & \\
\hline Ansiedade (escala HADS) & & & & & & & \\
\hline Moderada & 24 & 26,7 & $20,5(5,1)$ & 0,13 & 10 & 41,7 & 0,088 \\
\hline Ligeira & 11 & 12,2 & $23,1(3,4)$ & 0,093 & 3 & 27,3 & 0,029 \\
\hline Ausente & 55 & 61,1 & $22,5(4,3)$ & & 10 & 18,2 & \\
\hline
\end{tabular}


Para determinar o valor preditivo dos índices ómega-3 e razão ómega-6 / ómega-3 no desempenho cognitivo utilizaram-se vários modelos de regressão linear múltipla, ajustando para idade/sexo e possíveis variáveis confundidoras (associadas ao desempenho, $p<0,1$ ); num primeiro modelo ajusta-se para o perfil sócio-demográfico e funcionalidade, no segundo modelo ajusta-se também para comorbilidades e medicação e no último modelo acrescenta-se ainda o consumo alimentar. Adoptou-se um valor de $p<0,05$ como limite superior para rejeitar erradamente a hipótese nula. A análise dos dados foi feita usando o IBM SPSS Statistics 21.

\section{RESULTADOS}

\section{Perfil dos participantes}

Dos 119 utentes elegíveis, foram excluídos 21, três dos quais devido ao abuso de consumo de álcool. Após a avaliação inicial foram ainda excluídos oito participantes, um deles não completou o questionário e os restantes por se ter confirmado serem analfabetos e o MoCA não ser adequado para distinguir o compromisso cognitivo neste estrato populacional $(7,2 \%$ do total da amostra). Os 90 participantes tinham idades entre os 55 e 82 anos $(m=64,0$; $d p=5,6)$ e os homens $(48,9 \%)$ tinham uma idade média superior $(65,7 ; d p=6,0$ vs 62,$4 ; d p=4,8)$. A maioria dos participantes $(71,1 \%)$ tinha quatro anos de escolaridade, oito $(8,8 \%)$ tinham nove anos e cinco $(5,5 \%) 14$ ou mais anos, valores semelhantes para os dois géneros. Cerca de $62 \%$ encontrava-se em situação de pré-reforma ou reforma e $22(24,4 \%)$ estavam ainda activos (Tabela 1). A região é predominantemente industrial, $42(46,7 \%)$ dos participantes exercem ou exerceram neste ramo de actividades e $25,6 \%$ são trabalhadores não qualificados ou não auferindo remuneração, incluindo aqui as $16(34,8 \%)$ mulheres, donas de casa. Quase todos os participantes vivem com a família (93,3\%), mantêm-se em actividade $(77,8 \%)$ e são independentes para as actividades de vida diária $(92,2 \%)$. A hipertensão arterial é o factor de risco vascular mais prevalente $(51,1 \%)$, seguido da dislipidemia $(44,4 \%)$, doença cardíaca $(26,7 \%)$ e diabetes $(17,8 \%)$ - $36(40,0 \%)$ têm mais de um factor de risco; as doenças neurológicas $(10,0 \%)$ ou cerebrovascular $(3,3 \%)$ são menos prevalentes (Tabela 2). Cerca de 32,2\% dos participantes têm antecedentes de depressão, embora apenas $11,1 \%$ estejam actualmente medicados com antidepressivos, proporção que aumenta para $31,1 \%$ na medicação com benzodiazepinas.

Os valores do índice ómega-3 variam entre 2,90 e 9,79 (média $=6,20 ; d p=1,50$ ) e da razão ómega-6 $/$ ómega-3 entre 1,14 e 2,95 (média = 2,12; dp = 0,39), a distribuição conjunta está representada na Fig. 2. Note-se que existe uma correlação linear entre os dois índices $(r=-0,65 ; p$ $<0,001$ ) que aumenta para $r=-0,82$ quando excluídos 2 valores extremos na combinação (valores baixos nos dois índices). Na Tabela 3 está descrito resumidamente o perfil de alimentação dos participantes, que se caracteriza por um alto consumo (mais de 4 vezes/semana) de legumes $(81,1 \%)$, lacticínios $(56,7 \%)$ e carne branca $(51,1 \%)$ en- quanto o peixe gordo $(68,9 \%)$, peixe magro $(64,4 \%)$, bacalhau $(55,6 \%)$, carne vermelha $(35,6 \%)$ e o peixe de conserva $(32,2 \%)$ são mais frequentemente consumidos 2 - 3 vezes/semana. Por outro lado os fumados, frutos secos e cefalópodes não fazem parte da alimentação de respectivamente $36,7 \%, 35,6 \%$ e $34,4 \%$ dos participantes. $O$ índice ómega-3 e a razão ómega-6 / ómega-3 estão correlacionados com a frequência do consumo de peixe magro $(r=0,26$ e $r=-0,25$, respetivamente) e de cefalópodes $(r=0,25$ e $r=-0,22$, respetivamente); o consumo de frutos secos está também correlacionado com o índice ómega-3 $(r=0,24)$ e a razão ómega- 6 / ómega- 3 com o consumo de lacticínios $(r=-0,26)$. A frequência de consumo dos restantes grupos alimentares descritos na Tabela 3 não está correlacionada com nenhum dos índices.

\section{Desempenho cognitivo}

A avaliação cognitiva com o MoCA variou entre 10 e 29 (média $=22,0 ; d p=4,5$ ) seguindo uma distribuição normal $(p>0,22)$. Os valores médios foram significativamente diferentes para os três níveis de educação $(p<0,05)$, para o sector de actividade profissional (quadros /empresários em comparação com os trabalhadores não qualificados, $p<0,05$ ) e segundo a funcionalidade (Tabela 1). Há também uma tendência linear na descida da pontuação com a idade $(p<0,02)$ e com o nível de ansiedade $(p<0,1)$. De um modo geral existe um pior desempenho cognitivo nos participantes com comorbilidades, embora só na presença de antecedentes de doença cerebrovascular, cardíaca ou hipertensão haja diferenças significativas (Tabela 2). O desempenho cognitivo diminui com o número de factores de risco vascular $(p<0,003)$, melhorando, embora não significativamente, nos participantes medicados com antidepressivos ou benzodiazepinas.

Um valor alto no índice ómega-3 $(p<0,002)$ ou um valor baixo na razão ómega- 6 / ómega-3 $(p<0,02)$ aumentam significativamente o desempenho cognitivo, mas ao considerar o modelo ANOVA com os dois factores e interacção, existe um efeito significativo do índice ómega-3 $(F(1,86)=$ $7,5 ; p<0,008)$ e interação $(F(1,86)=7,1 ; p<0,009)$ e não da razão ómega- 6 / ómega-3, indicando que este aumento significativo se reporta ao subgrupo com uma razão baixa, vindo do contraste entre um n-3 alto e baixo (Tabela 2). Os grupos alimentares cujo consumo está associado ao desempenho cognitivo são os cefalópodes, peixe em conserva e frutos secos; um aumento na frequência do consumo destes alimentos traduz-se numa melhoria do desempenho cognitivo (Tabela 3).

Independentemente das variáveis que possam integrar o modelo de regressão, os participantes com um valor de ómega-3 acima da média e uma razão n-6 / n-3 $\leq 2$ têm em média uma pontuação no MoCA 1-2 valores superior aos restantes e os participantes com um valor de ómega-3 abaixo da média e uma razão n- 6 / n-3 $\leq 2$ têm em média uma pontuação no MoCA 5-6 valores inferior aos restantes (Tabela 4). Os outros preditores independentes do desempenho cognitivo são a educação $(p<0,001)$, o número de 
Tabela 2 - Descrição dos participantes, desempenho cognitivo (MoCA) e prevalência de compromisso cognitivo segundo comorbilidades, medicação e avaliação laboratorial

\begin{tabular}{|c|c|c|c|c|c|c|c|}
\hline \multirow{2}{*}{ Características } & \multicolumn{2}{|c|}{ Participantes } & \multicolumn{2}{|c|}{ Avaliação cognitiva } & \multicolumn{3}{|c|}{ Compromisso cognitivo } \\
\hline & $\mathrm{n}$ & $\%$ & Média (dp) & $p \dagger$ & $\mathrm{n}$ & Prevalência (\%) & $p \neq$ \\
\hline \multicolumn{8}{|c|}{ Doença cerebrovascular } \\
\hline Sim & 3 & 3,3 & $15,3(4,7)$ & 0,008 & 2 & 66,7 & 0,16 \\
\hline Não & 87 & 96,7 & $22,2(4,3)$ & & 21 & 24,1 & \\
\hline \multicolumn{8}{|l|}{ Doença cardíaca } \\
\hline Sim & 24 & 26,7 & $20,4(5,2)$ & 0,037 & 7 & 29,2 & 0,63 \\
\hline Não & 66 & 73,3 & $22,6(4,1)$ & & 16 & 24,2 & \\
\hline \multicolumn{8}{|l|}{ Diabetes } \\
\hline Sim & 16 & 17,8 & $20,4(5,5)$ & 0,12 & 5 & 31,3 & 0,56 \\
\hline Não & 74 & 82,2 & $22,3(4,2)$ & & 18 & 24,3 & \\
\hline \multicolumn{8}{|l|}{ Hipertensão arterial } \\
\hline Sim & 46 & 51,1 & $20,8(4,9)$ & 0,009 & 16 & 34,8 & 0,040 \\
\hline Não & 44 & 48,9 & $23,3(3,6)$ & & 7 & 15,9 & \\
\hline \multicolumn{8}{|l|}{ Dislipidemia } \\
\hline Sim & 40 & 44,4 & $21,8(5,2)$ & 0,70 & 11 & 27,5 & 0,71 \\
\hline Não & 50 & 55,6 & $22,2(3,9)$ & & 12 & 24,0 & \\
\hline \multicolumn{8}{|c|}{$\mathrm{N}^{\circ}$ factores de risco vascular * } \\
\hline $0-1$ & 54 & 60,0 & $22,9(3,6)$ & 0,011 & 10 & 18,5 & 0,12 \\
\hline $2-3$ & 30 & 33,3 & $21,1(5,0)$ & & 10 & 33,3 & 0,045 \\
\hline 4 & 6 & 6,7 & $17,8(6,3)$ & & 3 & 50,0 & \\
\hline \multicolumn{8}{|c|}{ Doença neurológica ** } \\
\hline Sim & 9 & 10,0 & $20,3(4,4)$ & 0,24 & 3 & 33,3 & 0,57 \\
\hline Não & 81 & 90,0 & $22,2(4,9)$ & & 20 & 24,7 & \\
\hline \multicolumn{8}{|c|}{ Depressão (antecedentes) } \\
\hline Sim & 29 & 32,2 & $21,7(5,3)$ & 0,65 & 11 & 37,9 & 0,063 \\
\hline Não & 61 & 67,8 & $22,2(4,0)$ & & 12 & 19,7 & \\
\hline \multicolumn{8}{|c|}{ Antidepressivos (medicação) } \\
\hline Sim & 10 & 11,1 & $24,2(4,4)$ & 0,099 & 2 & 20,0 & 0,67 \\
\hline Não & 80 & 88,9 & $21,7(4,4)$ & & 21 & 26,3 & \\
\hline \multicolumn{8}{|c|}{ Benzodiazepinas (medicação) } \\
\hline Sim & 28 & 31,1 & $22,4(5,3)$ & 0,61 & 10 & 35,7 & 0,14 \\
\hline Não & 62 & 68,9 & $21,8(4,1)$ & & 13 & 21,0 & \\
\hline \multicolumn{8}{|l|}{ Índice ómega-3 } \\
\hline Baixo $(<6,2)$ & 48 & 53,3 & $20,5(4,8)$ & 0,002 & 16 & 33,3 & 0,071 \\
\hline Alto $(\geq 6,2)$ & 42 & 46,7 & $23,6(3,5)$ & & 7 & 16,7 & \\
\hline \multicolumn{8}{|c|}{ Razão ómega-6 / ómega-3 } \\
\hline Alta $(>2,0)$ & 52 & 57,8 & $21,0(4,3)$ & 0,016 & 14 & 26,9 & 0,73 \\
\hline Baixa $(\leq 2,0)$ & 38 & 42,2 & $23,3(4,5)$ & & 9 & 23,7 & \\
\hline \multicolumn{8}{|c|}{ Combinação razão/ ómega-3 } \\
\hline Baixa/Baixo & 6 & 6,7 & $18,0(6,3)$ & & 5 & 83,3 & \\
\hline Baixa/Alto & 32 & 35,5 & $24,3(3,3)$ & ค คn:a & 4 & 12,5 & 0,004 \\
\hline Alta/Alto & 10 & 11,1 & $21,1(3,2)$ & & 3 & 30,0 & \\
\hline Alta/Baixo & 42 & 46,7 & $21,0(4,5)$ & & 11 & 26,2 & \\
\hline
\end{tabular}


Tabela 3 - Descrição dos participantes, desempenho cognitivo (MoCA) e prevalência de compromisso cognitivo segundo a frequência do consumo de grupos de alimentos

\begin{tabular}{|c|c|c|c|c|c|c|c|}
\hline \multirow{2}{*}{ Grupo de alimentos } & \multicolumn{2}{|c|}{ Participantes } & \multicolumn{2}{|c|}{ Avaliação cognitiva } & \multicolumn{3}{|c|}{ Compromisso cognitivo } \\
\hline & $\mathrm{n}$ & $\%$ & Média (dp) & $p \dagger$ & $\mathrm{n}$ & Prevalência (\%) & $p \ddagger$ \\
\hline Peixe magro & & & & 0,62 & & & 0,20 \\
\hline$\leq 1 \mathrm{vez} /$ semana & 26 & 28,9 & $21,7(4,3)$ & 0,45 & 9 & 34,6 & 0,091 \\
\hline 2 - 3 vezes / semana & 58 & 64,4 & $22,0(4,7)$ & & 14 & 24,1 & \\
\hline$\geq 4$ vezes / semana & 6 & 6,7 & $23,7(3,2)$ & & 0 & 0,0 & \\
\hline Peixe gordo & & & & 0,92 & & & 0,17 \\
\hline$\leq 1 \mathrm{vez} /$ semana & 22 & 24,4 & $22,1(4,0)$ & 0,93 & 8 & 36,4 & 0,075 \\
\hline 2 - 3 vezes / semana & 62 & 68,9 & $21,9(4,8)$ & & 15 & 24,2 & \\
\hline$\geq 4$ vezes / semana & 6 & 6,7 & $22,7(3,4)$ & & 0 & 0,0 & \\
\hline Cefalópodes & & & & 0,013 & & & 0,044 \\
\hline Nunca / raramente & 39 & 43,3 & $20,4(5,0)$ & 0,009 & 15 & 38,5 & 0,052 \\
\hline 1 vez / mês - 1 vez / semana & 30 & 33,3 & $23,2(3,1)$ & & 4 & 13,3 & \\
\hline$\geq 2$ vezes / semana & 21 & 23,3 & $23,2(4,3)$ & & 4 & 19,0 & \\
\hline Bacalhau & & & & 0,25 & & & 0,037 \\
\hline Nunca / raramente & 7 & 7,8 & $20,6(4,7)$ & 0,66 & 4 & 57,1 & 0,85 \\
\hline $1 \mathrm{vez}$ / mês - $1 \mathrm{vez}$ / semana & 31 & 34,4 & $23,0(3,9)$ & & 4 & 12,9 & \\
\hline$\geq 2$ vezes / semana & 52 & 57,8 & $21,6(4,7)$ & & 15 & 28,2 & \\
\hline Peixe em conserva & & & & 0,089 & & & 0,15 \\
\hline Nunca / raramente & 28 & 31,1 & $20,6(5,5)$ & 0,028 & 10 & 35,7 & 0,058 \\
\hline 1 vez / mês - 1 vez / semana & 28 & 31,1 & $22,0(4,3)$ & & 8 & 28,6 & \\
\hline$\geq 2$ vezes / semana & 34 & 37,8 & $23,2(3,4)$ & & 5 & 14,7 & \\
\hline Carne branca & & & & 0,61 & & & 0,68 \\
\hline$\leq 1 \mathrm{vez} /$ semana & 6 & 6,7 & $23,2(4,2)$ & 0,33 & 2 & 33,3 & 0,76 \\
\hline 2 - 3 vezes / semana & 38 & 42,2 & $22,3(5,2)$ & & 8 & 21,1 & \\
\hline$\geq 4$ vezes / semana & 46 & 51,1 & $21,6(3,8)$ & & 13 & 28,3 & \\
\hline Carne vermelha & & & & 0,59 & & & 0,46 \\
\hline$\leq 1 \mathrm{vez} /$ semana & 33 & 36,7 & $22,6(4,3)$ & 0,31 & 6 & 18,2 & 0,36 \\
\hline 2 - 3 vezes / semana & 32 & 35,5 & $21,9(5,1)$ & & 10 & 31,3 & \\
\hline$\geq 4$ vezes / semana & 25 & 27,8 & $21,4(3,9)$ & & 7 & 28,0 & \\
\hline Fumados & & & & 0,59 & & & 0,12 \\
\hline Nunca / raramente & 46 & 51,1 & $21,7(4,5)$ & 0,90 & 10 & 21,7 & 0,11 \\
\hline 1 vez / mês - 1 vez / semana & 29 & 32,2 & $22,7(4,2)$ & & 6 & 20,7 & \\
\hline$\geq 2$ vezes / semana & 15 & 16,7 & $21,5(5,0)$ & & 7 & 46,7 & \\
\hline Frutos secos* & & & & 0,005 & & & 0,070 \\
\hline Nunca / raramente & 60 & 66,7 & $21,0(4,3)$ & 0,022 & 19 & 31,7 & 0,23 \\
\hline $1 \mathrm{vez} /$ mês - $1 \mathrm{vez} / \mathrm{semana}$ & 19 & 21,1 & $24,7(3,5)$ & & 1 & 5,3 & \\
\hline$\geq 2$ vezes / semana & 11 & 12,2 & $22,6(5,3)$ & & 3 & 27,3 & \\
\hline Lacticínios & & & & 0,69 & & & 0,95 \\
\hline$\leq 1 \mathrm{vez} /$ semana & 25 & 27,8 & $21,4(4,6)$ & 0,39 & 6 & 24,0 & 0,92 \\
\hline 2 - 3 vezes / semana & 14 & 15,5 & $22,0(4,6)$ & & 4 & 28,6 & \\
\hline$\geq 4$ vezes / semana & 51 & 56,7 & $22,3(4,4)$ & & 13 & 25,5 & \\
\hline Legumes & & & & 0,55 & & & 0,69 \\
\hline$<4$ vezes / semana & 17 & 18,9 & $21,4(4,4)$ & & 5 & 29,4 & \\
\hline$\geq 4$ vezes / semana & 73 & 81,1 & $22,1(4,5)$ & & 18 & 24,7 & \\
\hline
\end{tabular}

$† 1^{\mathrm{a}}$ linha teste para comparação de médias e $2^{\mathrm{a}}$ linha teste de tendência linear nas médias; $\ddagger 1^{\mathrm{a}}$ linha teste para comparação de proporções e $2^{\mathrm{a}}$ linha teste de tendência linear nas proporções. * Variável em que existe uma relação quadrática significativa $(p<0,05)$ na variação do desempenho 
Compromisso cognitivo

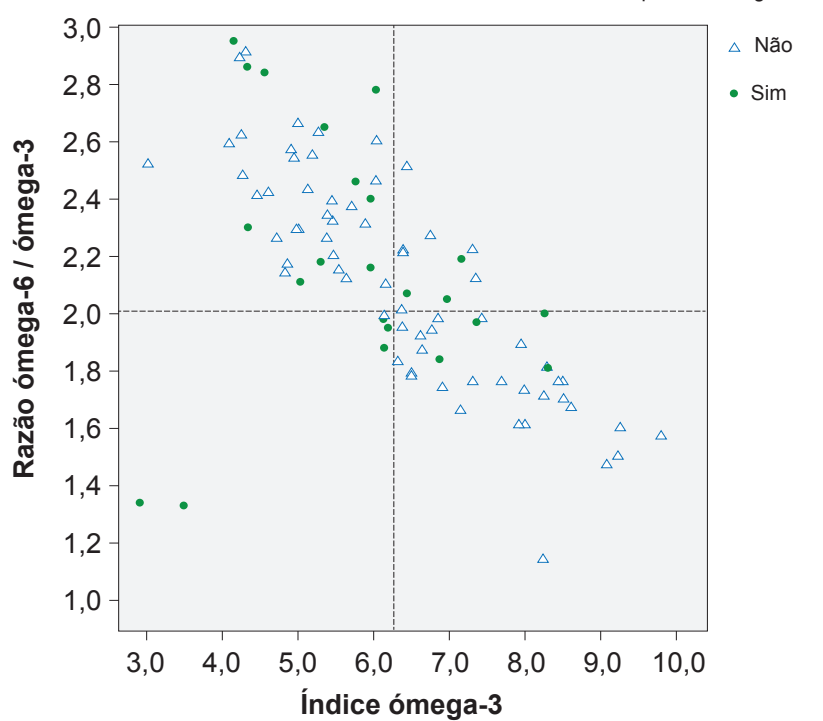

Figura 2 - Diagrama de dispersão da razão ómega 6 / ómega 3 no índice ómega-3

factores de risco vascular $(p<0,02)$ e a frequência do consumo de frutos secos. Dependendo do número de factores incluídos no modelo a inclusão das variáveis laboratoriais resulta num aumento relativo do $\mathrm{R}^{2}$ em 45,6\% (Modelo 1), 28,4\% (Modelo 2) e 19,9\% (Modelo 3).

\section{Compromisso cognitivo}

Dos 90 participantes, 23 tinham um valor no MoCA inferior ao valor de referência, uma prevalência de 25,6\% (IC 95\%: 16,4 - 34,7\%), 18,2\% (IC 95\%: 6,3 - 30,0\%) em homens e 32,6\% (IC 95\%: 18,5 - 46,7\%) em mulheres (Tabela 1). A prevalência atinge um valor de $66,7 \%$ nos participan- tes com 1 - 3 anos de escolaridade, diminuindo para 18,8\% e $21,4 \%$ nas escolaridades mais altas $(p<0,014)$. A prevalência é mais alta nos participantes dependentes $(71,4 \%)$ e aumenta linearmente com o grau de depressão $(p<0,03)$ e ansiedade $(p<0,03)$; é tendencialmente mais alta nos participantes com comorbilidades, em particular nos hipertensos $(34,8 \%$ vs $15,9 \%, p<0,04)$, e aumenta com o número de factores de risco vascular $(p<0,05)$ (Tabela 2). Embora a prevalência de compromisso cognitivo seja mais alta nos participantes com ómega-3 mais baixo e razão n-6 / n-3 alta, a diferença reporta-se ao estrato com uma razão n-6 / n-3 baixa contrastando aqueles que têm um valor ómega-3 baixo com uma prevalência de $83,3 \%$ e ómega-3 alto com uma prevalência de $12,5 \%$ (Fig. 2). A prevalência não está associada à frequência do consumo alimentar, embora seja tendencialmente mais baixa quando o consumo de peixe aumenta, gordo, magro, em conserva, bacalhau ou mesmo cefalópodes, contrariamente ao que acontece com a frequência do consumo de carne, em especial fumados (Tabela 3).

Considerando o compromisso cognitivo nos vários domínios do MoCA, observamos que este é mais frequente no que respeita à Linguagem $(46,7 \%)$, Memória $(40,0 \%)$ e Abstracção (36,7\%) (Tabela 5). Os valores nos índices ómega-3 e razão n-6 / n-3 estão associados a um compromisso na Nomeação $(p<0,003)$ e na Memória $(p<0,002)$ e não aos outros domínios.

Em relação aos resultados obtidos na bateria de testes neuropsicológicos e considerando o compromisso cognitivo nos outros testes neuropsicológicos, este é mais frequente na Memória (74,4\%), Linguagem (25,6\%) e Atenção e Velocidade de Processamento (24,4\%). O compromisso cognitivo na Atenção e Velocidade de Processamento está

Tabela 4 - Resultados dos modelos de regressão linear da avaliação cognitiva nas características associadas

\begin{tabular}{|c|c|c|c|c|c|c|}
\hline \multirow{2}{*}{ Variáveis } & \multicolumn{2}{|c|}{ Modelo 1} & \multicolumn{2}{|c|}{ Modelo 2} & \multicolumn{2}{|c|}{ Modelo 3} \\
\hline & B & $p$ & B & $p$ & B & $p$ \\
\hline Género (feminino versus masculino) & $-0,97$ & 0,24 & $-0,85$ & 0,29 & $-1,11$ & 0,18 \\
\hline Idade (anos) & $-0,18$ & 0,012 & $-0,14$ & 0,052 & $-0,10$ & 0,15 \\
\hline Educação (anos) & 0,49 & 0,001 & 0,49 & 0,001 & 0,42 & 0,001 \\
\hline Sector profissional (quadros versus outros) & 0,44 & 0,70 & 0,46 & 0,67 & 0,71 & 0,51 \\
\hline Funcionalidade (dependente versus independente) & $-1,95$ & 0,18 & $-1,58$ & 0,26 & $-1,47$ & 0,28 \\
\hline Ansiedade (escala HADS) & & & $-0,14$ & 0,083 & $-0,08$ & 0,35 \\
\hline $\mathrm{N}^{\circ}$ factores risco vascular & & & $-0,66$ & 0,035 & $-0,74$ & 0,014 \\
\hline Toma antidepressivos (sim versus não) & & & 2,51 & 0,032 & 2,05 & 0,075 \\
\hline Consumo de frutos secos (CFS) & & & & & 1,65 & 0,013 \\
\hline $\mathrm{CFS}^{2}$ & & & & & $-0,25$ & 0,030 \\
\hline Consumo de peixe de conserva & & & & & 0,31 & 0,11 \\
\hline Consumo de cefalópodes & & & & & $-0,11$ & 0,60 \\
\hline$n-3$ alto e razão n-6 / n-3 baixa versus outros & 1,98 & 0,022 & 1,91 & 0,021 & 1,59 & 0,048 \\
\hline n-3 baixo e razão n-6 / n-3 baixa versus outros & $-5,80$ & 0,001 & $-5,10$ & 0,002 & $-5,03$ & 0,002 \\
\hline $\mathrm{R}^{2}(\%)$ * & 31,8 & 46,3 & 41,5 & 53,3 & 49,2 & 59,0 \\
\hline
\end{tabular}

* $1^{\mathrm{a}}$ coluna $\mathrm{R}^{2}$ do modelo sem os valores laboratoriais e na $2^{\mathrm{a}}$ coluna $\mathrm{R}^{2}$ do modelo completo 
Tabela 5 - Associação entre o índice ómega-3 e a razão ómega-6 / ómega 3 e compromisso em domínios específicos

\begin{tabular}{|c|c|c|c|c|c|c|}
\hline \multirow[b]{2}{*}{ Domínios } & \multicolumn{2}{|c|}{ Participantes com défice } & \multicolumn{3}{|c|}{ Valores laboratoriais } & \multirow[b]{2}{*}{$p$} \\
\hline & $\mathbf{n}$ & $\%$ & $\begin{array}{c}n-6 / n-3 \leq 2 \\
\text { e } n-3>6,2 \\
(n=32)\end{array}$ & $\begin{array}{c}n-6 / n-3>2 \\
(n=52)\end{array}$ & $\begin{array}{c}n-6 / n-3 \leq 2 \\
\text { e } n-3<6,2 \\
(n=6)\end{array}$ & \\
\hline \multicolumn{7}{|l|}{ MoCA global e domínios } \\
\hline MoCA & 23 & 25,6 & $4(12,5)$ & $14(26,9)$ & $5(83,3)$ & 0,001 \\
\hline Visuo-espacial / Executiva & 20 & 22,2 & $5(15,6)$ & $13(25,0)$ & $2(33,0)$ & 0,48 \\
\hline Nomeação & 20 & 22,2 & $1(3,1)$ & $16(30,8)$ & $3(50,0)$ & 0,003 \\
\hline Memória & 36 & 40,0 & $6(18,8)$ & $25(48,1)$ & $5(83,3)$ & 0,002 \\
\hline Atenção & 6 & 6,7 & $2(6,3)$ & $3(5,8)$ & $1(16,7)$ & 0,59 \\
\hline Linguagem & 42 & 46,7 & $14(43,8)$ & $23(44,2)$ & $5(83,3)$ & 0,18 \\
\hline Abstracção & 33 & 36,7 & $8(25,0)$ & $23(44,2)$ & $2(36,7)$ & 0,20 \\
\hline \multicolumn{7}{|l|}{ Domínios teste neuropsicológicos } \\
\hline Atenção e Velocidade de Processamento & 22 & 24,4 & $1(3,1)$ & $18(34,6)$ & $3(50,0)$ & 0,002 \\
\hline Memória & 67 & 74,4 & $21(65,6)$ & $40(76,4)$ & $6(100)$ & 0,17 \\
\hline Funcionamento Executivo & 13 & 14,4 & $0(0,0)$ & $11(21,2)$ & $2(33,3)$ & 0,011 \\
\hline Linguagem & 23 & 25,6 & $5(15,6)$ & $14(26,9)$ & $4(66,7)$ & 0,030 \\
\hline
\end{tabular}

Nota: No domínio Orientação do MoCA todos os participantes têm provas normais

n-3: ómega-3; n-6: ómega-6

associado aos valores dos índices $(p<0,002)$ e tendencialmente também o Funcionamento Executivo e Linguagem. O grupo de participantes com um índice ómega-3 $>6,2$ e uma razão n-6 / n-3 $\leq 2$ tem menos compromisso, seguido do grupo com uma razão n- $6 / \mathrm{n}-3>2$ (independentemente do valor de n-3) e valores de ómega-3 $<6,2$ e razão n- 6 / n-3 $\leq 2$ são indicativos de maior compromisso nos domínios citados (resultados não mostrados).

\section{DISCUSSÃO}

Pode concluir-se que nesta população com mais de 55 anos de idade do concelho de Lousada existe uma relação entre os valores laboratoriais de n-3 e n-6 / n-3 e o desempenho cognitivo. Em particular o estrato populacional com n-3 abaixo da média amostral e razão n- $6 / n-3>2$ tem um elevado risco de défice cognitivo enquanto que o estrato com valores de $\mathrm{n}-3$ acima da média amostral e razão n-6 / n-3 $\leq 2$ é o mais protegido de compromisso cognitivo. Este compromisso avaliado pelo MoCA reporta-se essencialmente a falhas na Nomeação e Memória, enquanto nos testes neuropsicológicos o compromisso é mais marcado na Atenção e Velocidade de Processamento e Funcionamento Executivo.

Poder-se-ia esperar que este desempenho e compromisso cognitivo se focasse nos estratos com valores da razão n-6 / n-3 altos, normalmente descritos com tendo um risco elevado de compromisso cognitivo. ${ }^{49}$ No entanto nesta população os valores da razão não excedem 3.0. A pouca variabilidade (limites: $1,14-2,95$ ) neste índice talvez possa ser explicada pela alimentação essencialmente de tipo mediterrânico, com consumo frequente de vegetais, carnes de animais criados ao ar livre e consumo de peixe.

As fontes de gorduras na alimentação apesar de mais diversificadas na Europa do que nos Estados Unidos, têm mudado muito nas últimas décadas, com maior consumo de óleo de girassol em detrimento do azeite, mesmo em países produtores como Portugal e Espanha. ${ }^{50}$

Olhando para a relação entre os valores dos índices e o desempenho cognitivo depois de ajustar para possíveis variáveis confundidoras, cerca de $10 \%$ da variabilidade é explicada pela existência destes dois estratos populacionais - valores no MoCA mais baixos nos participantes com simultaneamente $n-3$ e n- 6 / $n-3$ baixos e valores no MoCA mais altos nos participantes com n-3 acima da média e razão n-6 / n-3 baixa. As restantes variáveis explanatórias do desempenho cognitivo com um efeito protector são a educação e a frequência do consumo de frutos secos, notando-se que nesta última o efeito protector se refere a um consumo moderado em comparação com os restantes (modelo de regressão com termo linear e quadrático ajustando a relação descrita na Tabela 3). Por outro lado o desempenho cognitivo é pior nos participantes com um maior número de factores de risco vascular. Estes factores têm já sido apontados na literatura pela sua associação com o desempenho cognitivo. $^{7}$

Beydoun e colaboradores relatam associação de compromisso num teste de fluência verbal com valores elevados de n-6 ácidos gordos, mesmo sem a razão n-6 / n-3 elevada. ${ }^{51}$ Num estudo envolvendo trezentos e quatro idosos institucionalizados os autores encontraram associação entre a razão n-6 / n-3 e declínio cognitivo avaliado pelo MMSE, em oposição ao consumo de peixe e de produtos marinhos. ${ }^{52}$ Outros estudos em humanos e animais suportam a noção de que a razão n-6 / n-3 está associada com risco de demência e declínio cognitivo, e o menor consumo de n-3 poderá acelerar entre $10 \%$ e $80 \%$ o declínio cognitivo. ${ }^{19-24}$ 


\section{CONCLUSÃO}

Embora a dimensão amostral não permita tirar conclusões mais definitivas quanto à existência de compromisso cognitivo (23 em 90 participantes), este estudo preliminar é um dos primeiros a considerar um conjunto de características da população mais amplo, baseando-se não só na informação clínica do utente do Serviço Nacional da Saúde, vantagem de ter uma população enumerada para seleccionar aleatoriamente a amostra, mas também informação sobre aspectos menos comuns, como o consumo alimentar, e que se mostraram relevantes para estudar o desempenho cognitivo.

Resumindo, numa população com literacia predominantemente baixa ( $84,4 \%$ com $\leq 4$ anos de escolaridade), em que $40 \%$ têm dois ou mais factores de risco vascular, com um consumo alimentar característico de uma dieta mediterrânica, o desempenho e compromisso cognitivo estão relacionados com os valores do índice ómega-3 e da razão n-6 / n-3 nos eritrócitos, sugerindo que estes são biomarcadores importantes na prevenção da deterioração cognitiva.

Demonstra ainda a necessidade de reforçar a ingestão de ácidos gordos n-3, nomeadamente através do consumo de peixe, algas e frutos secos. Nesta população é ainda necessário reforçar as medidas de restrição de sal como

\section{REFERÊNCIAS}

1. Petersen RC, Smith GE, Waring SC, Ivnik RJ, Tangalos EG, Kokmen E. Mild cognitive impairment: clinical characterization and outcome. Arch Neurol. 1999;56:303-8.

2. Petersen RC. Mild cognitive impairment as a diagnostic entity. J Intern Med. 2004;256:183-94.

3. Proceedings of the 1st Key symposium: Mild Cognitive Impairment. Stockholm, Sweden, September 2003. J Intern Med. 2004;256:179-246.

4. Petersen RC, Morris JC. Mild cognitive impairment as a clinical entity and treatment target. Arch Neurol. 2005;62:1160-3.

5. Lopez OL, Jagust WJ, DeKosky ST, Becker JT, Fitzpatrick A, Dulberg $C$, et al. Prevalence and classification of mild cognitive impairment in the cardiovascular health study cognition: part 1. Arch Neurol. 2003;60:1385-9.

6. Feldman $\mathrm{HH}$, Jacova C. Mild cognitive impairment. Am J Geriatr Psychiatry. 2005;13:645-55.

7. Nunes B, Silva RD, Cruz VT, Roriz JM, Pais J, Silva MC. Prevalence and pattern of cognitive impairment in rural and urban populations from Northern Portugal. BMC Neurology. 2010;11:10-42.

8. van Gelder BM, Tijhuis M, Kalmijn S, Kromhout D. Fish consumption, $\mathrm{n}-3$ fatty acids, and subsequent $5-\mathrm{y}$ cognitive decline in elderly men: the Zutphen Elderly Study. Am J Clin Nutr. 2007;85:1142-7.

9. Jicha GA, Markesbery WR. Omega-3 fatty acids: potential role in the management of early Alzheimer's disease. Clin Interv Aging. 2010;5:4561.

10. Roberts RO, Geda YE, Cerhan JR, Knopman DS, Cha RH, Christianson $\mathrm{T}$, et al. Vegetables, unsaturated fats, moderate alcohol intake, and mild cognitive impairment. Dement Geriatr Cogn Disord. 2010;29:413-23.

11. Barak Y, Aizenberg D. Is dementia preventable? Focus on Alzheimer's disease. Expert Rev Neurother. 2010;10:1689-98.

12. Kamphuis PJ, Scheltens P. Can nutrients prevent or delay onset of Alzheimer's disease? J Alzheimers Dis. 2010;20:765-75.

13. Lopez LB, Kritz-Silverstein D, Barret Connor E. High dietary and plasma levels of the omega-3 fatty acid docosahexaenoic acid are associated with decreased dementia risk: the Rancho Bernardo study. J Nutr Health Aging. 2011;15:25-31.

14. Walker JG, Batterham PJ, Mackinnon AJ, Jorm AF, Hickie I, Fenech $\mathrm{M}$, et al. Oral folic acid and vitamin B-12 supplementation to prevent cognitive decline in community-dwelling older adults with depressive symptoms- the Beyond Ageing Project: a randomized controlled trial. Am J Clin Nutr. 2012;95:194-203. prevenção e controlo da hipertensão arterial, importante factor de risco associado ao declínio cognitivo.

\section{PROTECÇÃO DE PESSOAS E ANIMAIS}

Os autores declaram que os procedimentos seguidos estavam de acordo com os regulamentos estabelecidos pelos responsáveis da Comissão de Investigação Clínica e Ética e de acordo com a Declaração de Helsínquia da Associação Médica Mundial.

\section{CONFIDENCIALIDADE DOS DADOS}

Os autores declaram ter seguido os protocolos do seu centro de trabalho acerca da publicação de dados.

\section{CONFLITOS DE INTERESSE}

Os autores declaram não terem qualquer conflito de interesse relativamente ao presente artigo.

\section{FONTES DE FINANCIAMENTO}

Os autores declaram ter recebido da Angelini Farmacêutica o suporte financeiro para execução das entrevistas e avaliações neuropsicológicas, e a determinação analítica da razão ómega-6 / ómega-3 eritrocitária.

15. Harris WS. The omega- 3 index as a risk factor for coronary heart disease. Am J Clin Nut. 2008;87:1997S-2002.

16. Molfino A, Gioia G, Fanelli FR, Muscaritoli M. The role for dietary omega-3 fatty acids supplementation in older adults. Nutrients. 2014;6:4058-72.

17. Loef $\mathrm{M}$, Walach $\mathrm{H}$. The omega-6/omega-3 ratio and dementia or cognitive decline: a systematic review on human studies and biological evidence. J Nutr Gerontol Geriatr. 2013;32:1-23.

18. Hammen C. Stress and depression. Annu Rev Clin Psychol. 2005;1:293319.

19. Conquer JA, Tierney MC, Zecevic J, Bettger WJ, Fisher RH. Fatty acids analysis of blood plasma of patients with Alzheimer's disease, other types of dementia, and cognitive impairment. Lipids. 2000;35:1305-12.

20. Cherubin A, Andres-Lacueva C, Martin A, Lauretani F, lorio AD, Bartali $B$, et al. Low plasma N-3 fatty acids and dementia in older persons: the InCHIANTI study. J Geront Biol Sc Med Sci. 2007;62:1120-6.

21. Perez SE, Berg BM, Moore KA, He B, Counts SE, Fritz JJ, et al. DHA diet reduces $A D$ pathology in young APPswe/PS1deltaE9 transgenic mice: possible gender effects. J Neurosci Res. 2010;88:1026-40.

22. Oksman M, livonen $\mathrm{H}$, Hogyes $\mathrm{E}$, Amtul Z, Penke B, Leenders I, et al. Impact of different saturated fatty acid, polyunsaturated fatty acid and cholesterol containing diets on beta-amyloid accumulation in APP/PS1 transgenic mice. Neurobiol Dis. 2006;23:563-72.

23. Lebbadi M, Julien C, Phivilay A, Tremblay C, Emond V, Kang JX, et al. Endogenous conversion of omega- 6 into omega-3 fatty acids improves neuropathology in an animal model of Alzheimer's disease. J Alzheimers Dis. 2011;27:853-69.

24. Yurko-Mauro K, McCarthy D, Rom D, Nelson EB, Ryan AS, Blackwell $A$, et al. Beneficial effects of docosahexanoic acid on cognition in agerealted cognitive decline. Alzheimers Dement. 2010;6:456-64.

25. Thies F, Garry JM, Yaqoob P, Rerkasem K, Williams J, Shearman $\mathrm{CP}$, et al. Association of $\mathrm{n}-3$ polyunsaturated fatty acids with stability of atherosclerotic plaques: a randomised controlled trial. Lancet. 2003;361:477-85.

26. Sofi F, Giorgi G, Cesari F, Gori AM, Mannini L, Parisi G, et al. The atherosclerotic risk profile is affected differently by fish flesh with a similar EPA and DHA content but different $n-6 / n-3$ ratio. Asia Pac J Clin Nutr. 2013;22:32-40.

27. Mehmetoglu I, Yerlikaya FH, Kurban S, Polat H. Plasma 3-3 fatty acid levels negatively and 3-6 fatty acid levels positively associated with other cardiovascular risk factors including homocysteine in severe 
obese subjects. Asia Pac J Clin Nutr. 2012;21:519-25.

28. Babor TF, Higgins-Biddle JC, Saunders JB, Monteiro MG. The alcohol use disorders identification test: guideline for use in primary care. $2^{\text {nd }} \mathrm{ed}$. Geneva: WHO; 2001.

29. Ginó S, Mendes T, Ribeiro F, Mendonça A, Guerreiro M, Garcia C. Escala de queixas de memória. In Grupo de Estudos de Envelhecimento Cerebral e Demências, editores. Escala e Testes na demência. Lisboa: GEECD; 2008. p.117-20.

30. Schmand B, Jonker C, Hooijer C, Lindeboom J. Subjective memory complaints may announce dementia. Neurology. 1996;46:121-5.

31. Freitas S, Simões M, Alves L, Santana I. Montreal Cognitive Assessment (MoCA): normative study for the Portuguese population. J Clin Exp Neuropsychol. 2011;3:989-96.

32. Nasreddine Z, Philips NA, Bédirian V, Charbonneau S, Whitehead V, Collin I, et al. The Montreal Cognitive Assessment, MoCA: a brief screening tool for mild cognitive impairment. J Am Geriat Soc. 2005;53:695-9.

33. Santana I, Duro D, Freitas S, Alves L, Simões M. The clock drawing test: Portuguese norms, by age and education, for three different scoring systems. Arch Clin Neuropsychol. 2013;28:375-87.

34. Babins L, Slater ME, Whitehead V, Chertkow H. Can an 18-point clockdrawing scoring system predict dementia in elderly individuals with mild cognitive impairment? J Clin Exp Neuropsychol. 2008;30:173-86.

35. Cavaco S, Gonçalves A, Pinto C, Almeida E, Gomes F, Moreira I, et al. Semantic fluency and phonemic fluency: regression-based norms for the Portuguese population. Arch Clin Neuropsychol. 2013;28:262-71.

36. Strauss E, Sherman EM, Spreen O. Acompendium of neuropsychological tests: administration, norms, and commentary. $3^{\text {rd }}$ edition. New York: Oxford University Press; 2006.

37. Fernandes S. Stroop: teste de cores e palavras. $1^{\text {a }}$ edição. Lisboa: CEGOG-TEA; 2012.

38. Golden CJ, Freshwater SN. Stroop color and word test: a manual for clinical and experimental uses. Chicago: Stoelting Co; 2002.

39. Brandt J. The Hopkins verbal learning test: development of a new verbal memory test with six equivalent forms. Clinical Neuropsychologist. 1991;5:125-42.

40. Wechsler D. Escala de memória de Wechsler (WMS-III). Lisboa: CEGOG-TEA; 2008

41. Wechsler D. Escala de inteligência de Wechsler para adultos (WAIS-III).
Lisboa: CEGOG-TEA; 2008.

42. Cavaco S, Gonçalves A, Pinto C, Almeida E, Gomes F, Moreira I, et al. Trail Making Test: regression-based norms for the Portuguese population. Arch Clin Neuropsychol. 2013;28:189-98.

43. Rabin LA, Barr WH, Burton LA. Assessment practices of clinical neuropsychologists in the United States and Canada: a survey of INS, NAN, and APA Division 40 members. Arch Clin Neuropsychol. 2005;20:33-65.

44. Madureira S, Verdelho A. Escala de actividades instrumentais de vida diária (IADL). In Mendonça A, Guerreiro M, Coords. Escala e testes na demência - Grupo de Estudos de Envelhecimento Cerebral e Demências. Lisboa: Novartis; 2007. p. 113-5.

45. Lawton MP, Brody EM. Assessment of older people: self-maintaining and instrumental activities of daily living. The Gerontologist. 1969;9:17986

46. Zigmond AS, Snaith RP. The hospital anxiety and depression scale. Acta Psychiat Scand. 1983;67:361-70.

47. Bandarra NM, Palma P, Batista I, Nunes ML, Branco P, Bruges M, et al. Supplementation of the diet of haemodialysis patients with portuguese canned sardines and evaluation of w3 fatty acid level in erythrocyte phospholipids. J AQUAT FOOD PROD T. 2004;13:61-8.

48. Janssen $\mathrm{Cl}$, Kiliaan AJ. Long-chain polyunsaturated fatty acids (LCPUFA) from genesis to senescence: the influence of LCPUFA on neural development, aging, and neurodegeneration. Progr Lipid Res. 2014:53:1-17.

49. Vercambre MN, Boutron-Ruault MC, Richie K, Clavel-Chapelon F, Berr C. Long-term association of food and nutrient intakes with cognitive and functional decline: a 13-year follow-up study of French women. $\mathrm{Br} J$ Nutr. 2009;102:419-27.

50. Sanders TA. Polyunsaturated fatty acids in the food chain in Europe. Am J Clin Nut. 2000;71:176S-8.

51. Beydoun MA, Kaufman JS, Sloan PD, Heiss G, Ibrahim J. n-3 Fatty acids, hypertension and risk of cognitive decline among older adults in the Atherosclerosis Risk in Communities (ARIC) study. Pub Health Nutr. 2008;11:17-29.

52. Gonzalez S, Huerta JM, Fernandez S, Patterson AM, Lasheras C. The relationship between dietary lipids and cognitive performance in an elderly population. Int J Food Sci Nutr. 2010;61:217-25. 